\title{
Design of Dust Concentration Monitoring System Based on Single Chip Microcomputer
}

\author{
Tao Lin \\ Nanjing Engineering Branch, Jiangsu Union Technical Institute, Nanjing, Jiangsu, 211135, China \\ ltnjgc@126.com
}

Keywords: Design and Research of Single Chip Microcomputer Dust Concentration Monitoring System.

\begin{abstract}
With the development of society, the improvement of economic level and the progress of science and technology, the industrial modern production technology of our country has been innovated constantly, but the dust produced in the actual industrial field production process will not only threaten the health of the staff, but also cause serious pollution to the atmospheric environment. Therefore, it is very important to carry out comprehensive monitoring of dust content in the industrial production environment, and the research and design of dust concentration monitoring system can use the infrared absorption dust monitoring system of single chip microcomputer to monitor the effect of dust concentration in real time and accurately. This article mainly aims at the microcomputer controller module circuit, the light source drive circuit, the amplifier filter circuit, the display module circuit design and so on related system hardware part, including the data collector, the analog-to-digital conversion control program, the upper computer program carries on the system design effect analysis.
\end{abstract}

\section{Introduction}

In the modern industrial production process, the dust actual production environment is more common derivatives. Especially in the production process of coal, chemical industry, mineral industry and other industrial industries, its environment is filled with a large number of dust particles, which can easily enter the human body's blood through the respiratory system. If the composition of the dust particles has harmful substances or radioactive elements, it is very easy to cause various diseases of the human body. Therefore, it is of great practical significance to monitor the concentration of dust in industrial production environment in real time to reduce the pollution of atmospheric environment and the threat of human health. Therefore, it is necessary to take effective measures to reduce dust pollution and improve the atmospheric environment, and also reduce the cost input caused by the impact of human health levels. From the 1980s 4 or 8 bit single chip microcomputer, developed to the current $300 \mathrm{M}$ high-speed single-chip microcomputer.

\section{Current Methods for Monitoring Dust Concentrations}

The main methods of dust concentration monitoring used in industrial production in China are sampling method and non-sampling method, which mainly refer to the technology of dust concentration detection in developed countries abroad. Sampling method mainly refers to the measurement of dust concentration by filtration weighing method, beta-ray method, piezoelectric vibration method and so on. The technical principle of this method is relatively simple, and the technical method is relatively low cost and the technical content is not high. However, sampling method is also relatively weak, such as low sensitivity and high periodicity requirement. And its accuracy is also very easy to be affected by factors such as temperature and humidity in the environment. The non-sampling method mainly includes light absorption method, light scattering method, triboelectric method and scintillation method. Compared with the sampling method, the non-sampling method has some technical difficulty, and the detection principle is more complex, 
but it has a high level of automation and real-time and continuous monitoring of dust concentration, and its accuracy is also high. Therefore, in the process of modern industrial production, the research on the monitoring method of dust concentration is mainly based on the light absorption method in the non-sampling method. [1].

\section{A Brief Introduction of Dust Concentration Monitoring System Based on Single Chip Microcomputer}

In the industrial production environment, the monitoring results of dust concentration are often affected by the factors such as temperature and humidity in the environment and the interference of light source, which makes the accuracy and accuracy of the monitoring results seriously reduced. Therefore, it is very important to design a monitoring system for dust concentration in industrial production environment. The design of the dust concentration monitoring system based on single chip microcomputer can effectively avoid and reduce the error caused by the interference of ambient temperature and humidity as well as the light source. The core of the technology lies in the advanced technical choice of the central processor to improve the stability of the whole system. After beam splitter processing into the industrial production environment and environmental detection object, using the detector infrared signal acquisition, and then through the amplifier filter circuit into the single chip microcomputer for further data analysis and data processing. In the system, the infrared detector is used as an intermediate conversion device for the conversion of infrared radiation signal to electrical signal, and it is also the core device of the dust concentration detection system, which is very important. The structure of the whole MCU dust concentration monitoring system is shown in the following figure:

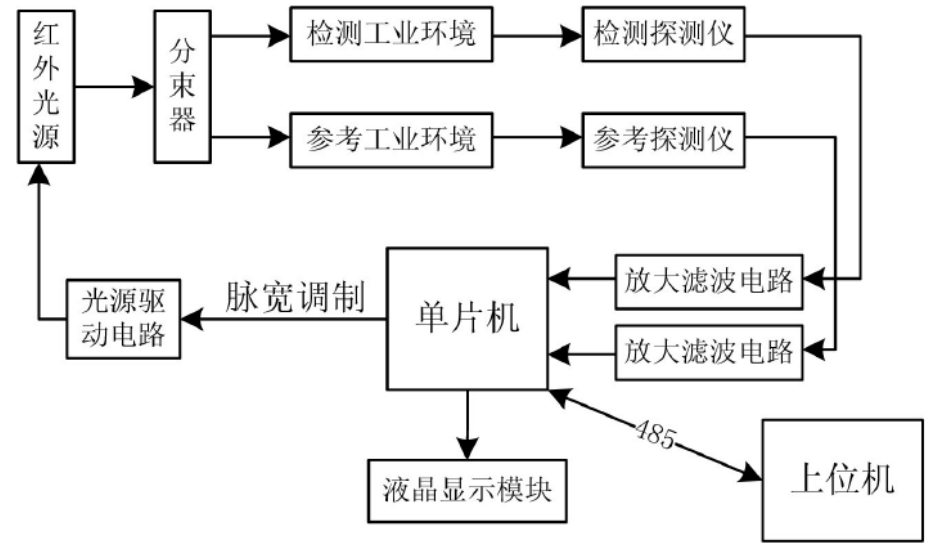

Figure 1 Structure diagram of dust concentration monitoring system based on single chip microcomputer

\section{Hardware Design of Dust Concentration Monitoring System Based on Single Chip Microcomputer}

\subsection{Thinking on the Design of Light Source Drive Circuit Module}

The hardware of the dust concentration monitoring system of single chip microcomputer. The design of the light source driving circuit module mainly uses the infrared light source, selects the corresponding stable type light-emitting diode, and uses the adjustable voltage regulator as the power supply source, as shown in Figure 2: 


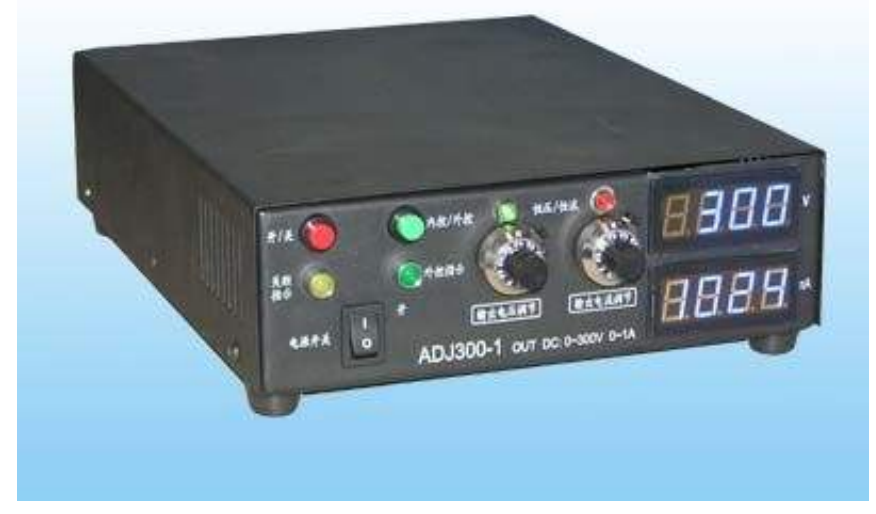

Figure 2 Adjustable DC regulator

A three-terminal adjustable positive regulator light-emitting diode is selected, and the output adjustable voltage is controlled between $1.2 \mathrm{~V}$ and $37 \mathrm{~V}$, and a thermal overload protection device and a short-circuit current limiting device are installed inside it, which can provide sufficient power support for the infrared light source as a stable power supply source.[2].

\subsection{Design and Analysis of Module Circuit of Microcontroller}

Considering the complexity and influence factors in the current industrial environment, the microcontroller selected in this paper is mainly MSP430F149 chip, which has strong advantages in operation performance and power consumption, and is a kind of microprocessor chip with excellent performance in instrument and instrument control products. The display selects the LCD series with higher performance displays. to display real-time monitoring information data.

\subsection{Ideas on Circuit Design of Amplifier Filter Module}

The circuit design of the amplifier filter module because the current of the detector output signal lamp is selected as the micro-security level, it is necessary to amplify the signal through the circuit of the amplifier filter module, so the selection of the blood chip needs to meet the requirements of low power consumption, low offset voltage, relatively small volume and less noise, so that it can effectively avoid the influence of different factors and complex environment in the industrial production environment. [3].

\section{Software Design of Dust Concentration Monitoring System Based on Single Chip Microcomputer}

\subsection{The Design Idea of the Software of the Modular Conversion Program}

The design of the software part is divided into data acquisition and analog-to-digital conversion as well as the program design of liquid crystal module. Usually, the main program design flow of the software part needs to be carried out according to the interrupt program data acquisition program, the serial communication program and the upper computer program when the software is processed. [4]. The result of the converted data is stored in the database. and make analysis treatment.

\subsection{Design Analysis of Data Collection Program}

The design of the data collection program is mainly completed in the interrupt process of the timer and passed. The data information is filtered and stored effectively, and when the timer enters the interrupt, the data acquisition instruction is executed according to the different interrupt timing time, and finally the data is stored and filtered and analyzed. In contrast, the design of data acquisition program is relatively simple, and its design process reference factors are not complex[5]. It should be noted that during the instruction execution of the data acquisition program, attention must be paid to the accurate setting of its timing time to improve the accuracy and accuracy of the 
dust concentration data collection. The design process is shown in Figure 3:

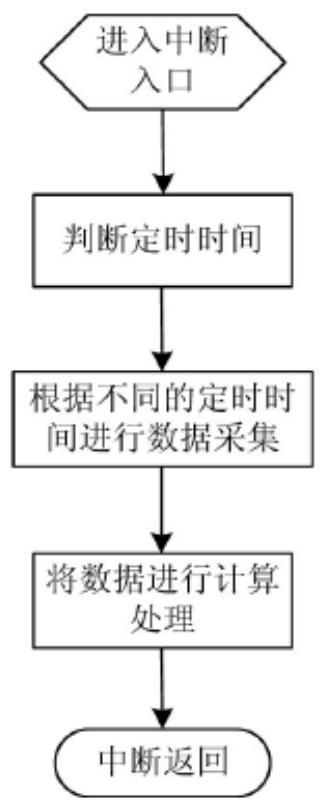

Figure 3 Data acquisition system software programming flow diagram

\subsection{Consideration on the Design of Upper Computer Program Software}

The upper computer program in the software program of dust concentration monitoring system is mainly transmitted through communication data. the upper computer interface of the dust concentration monitoring system is displayed for the measurement data information and the data chart information. In this way, the data of dust concentration can be clearly displayed, especially for the variation of dust concentration in different time and real-time. To enable the relevant technicians to carry out accurate data screening, and to express the data beyond the threshold of dust concentration in a more conspicuous color, so as to make a clear distinction between the data connections of different detection points, and then to realize the accurate analysis of the real-time monitoring data of dust concentration in the industrial environment, and to make a relative treatment plan on the basis of it [6].

\section{Conclusion}

With the continuous development of industrial economy and the continuous research of industrial science and technology, it is necessary to take necessary measures and advanced technology to monitor and detect the dust concentration in the industrial production environment in real time, improve the quality of industrial production environment, improve the safety of industrial production and life, eliminate the influence of dust concentration on industrial production, the threat to human health and the harm of air pollution. Furthermore, the accuracy and accuracy of the crossbeam and the experimental dust concentration measurement can be used to reduce the influence of dust pollution and improve the accuracy and stability of dust concentration monitoring in industrial production.

\section{Acknowledgement}

Jiangsu University "blue and green project" funded fund project (Su teacher (2017) No. 15)

\section{References}

[1] Li, Huan. Design of dust concentration monitoring system based on single chip microcomputer. Automation Technology and Applications , vol. 38, no. 06, pp. 113-116, 2019. 
[2] Zhao, Xinran.,, Li Qun., Wang, Weidong., Xu,. Zhiqiang, Lu, Hengrun. Design of mass overthe-line dust concentration monitoring system. China Testing, vol. 45, no. 04, pp. 84-88+132, 2019.

[3] Li, Bo., Hou, Jiaquan. Design of dust detection system based on single chip microcomputer control. Technological Innovation and Productivity, vol. 303, no. 04, pp. 54-56, 2019.

[4] Zhang, Yufeng., Huang, Keyan.,, Cao Jian. Simple single chip microcomputer based air dust and temperature and humidity detection system design. China Strategic Emerging Industries, vol. 172, no. 40, pp. 144, 2018.

[5] Xiao, Qiang., Xu, Dong., Wang, Xiaochao., Wan, Zaihong., Li, Xiangwen. Research on dust monitoring and alarm system based on single chip microcomputer. Wireless Internet Technology, no. 16, 2019.

[6] Ma, Dongning., Zhao, Pengfei., Wang, Zhenshuai., Tang, Yuan. An intelligent dust monitoring and alarm system based on single chip microcomputer. China High-Tech Enterprises, no. 5, 2017. 